\title{
Tratamiento conservador de fracturas mandibulares en pacientes pediátricos
}

Non-surgical treatment alternatives of mandibular fractures in pediatric patients

\section{Resumen}

Los traumas faciales en pacientes pediátricos presentan especiales consideraciones en lo que respecta a su prevalencia, diagnóstico y tratamiento, las cuales difieren de las encontradas en los adultos. La prevalencia de fracturas faciales en niños es baja, variando de 13 a $15 \%$, lo cual puede deberse tanto a factores anatómicos como ambientales, siendo las causas principales los accidentes de tránsito, caídas de propia altura y los accidentes deportivos.

La elección de la alternativa de tratamiento dependerá de las características intrínsecas de la fractura, la edad del paciente y de la etapa de dentición que éste presente. Esta heterogeneidad, unida a la baja incidencia de fracturas faciales en niños y la limitada experiencia de muchos clínicos y cirujanos en el manejo de traumas significativos en pacientes pediátricos puede justificar la falta de consenso en el tratamiento. En general, a menor edad del paciente, se tiende a un manejo conservador de la fractura.

Fracturas con mínimo desplazamiento pueden ser tratadas de manera conservadora, mediante fijación maxilomandibular, observación e indicación de dieta blanda y reposo o mediante medicación analgésica cuando fuera necesario. Fracturas que presenten de moderado a severo desplazamiento pueden necesitar de reducción abierta con fijación interna rígida.

\begin{abstract}
Facial traumas in pediatric patients show considerations that are not present in adults, related to prevalence, diagnosis and treatment. Facial fractures prevalence in children is low, ranging from 13 to $15 \%$. This can be due to anatomic or environmental factors, such as traffic accidents, falls and sport accidents as the main ones. The choice of treatment alternative will depend on the intrinsic characteristics of the fracture, the patient's age and teeth development stage.

Thus heterogeneity along with low incidence of facial fracture in pediatric patients, and limited experience of some clinical and surgical professionals in child trauma management can be support for lack of consensus in treatment. Generally, minor age of patient have more conservative management.

Fractures with minimum displacement can be treated in a conservative way, by means of máxilo mandibular fixation, observation, soft diet and rest or analgesic medication when necessary. The open reduction with rigid internal fixation should be performed only in cases of moderate to severe bone displacement.
\end{abstract}

\section{Introducción}

Las fracturas faciales en niños son relativamente raras si son comparadas con las fracturas faciales en adultos, siendo las de mandíbula las más frecuentes. Este hueso facial, debido a su topografía, anatomía y proyección en el tercio inferior de la cara, es frecuentemente afectado por traumas o por otro tipo de injurias (Filho 2000) ${ }^{(1)}$.

Las fracturas de mandíbula pediátrica pueden ser tratadas con una amplia variedad de métodos de fijación. Fracturas incompletas o levemente desplazadas, como las fracturas subcondilares, pueden ser tratadas con métodos tradicionales de reducción cerrada o mediante dieta blanda y un riguroso seguimiento. Fracturas con un moderado a severo desplazamiento, son mejor tratadas mediante la reducción abierta y fijación interna rígida mediante dispositivos metálicos o biodegradables de osteosíntesis (Eppley 1997) ${ }^{(3)}$. Como principios básicos, el grado de desplazamiento va a condicionar la necesidad de osteosíntesis, la fase de dentición determinará las técnicas de inmovilización y de osteosintesis a emplear $y$, la localiza-

\section{José Muñante-Cárdenas'; Sergio Olate $^{2}$, Miguel Jaimes ${ }^{3}$; Erick Gonzales del Castillo4, José Ricardo de Alberga- ria-Barbosa ${ }^{5}$}

Cirujano-Dentista.

Cirujano-Dentista. Profesor de la facultad de Medicina, Universidad de la Frontera, Temuco-Chile.

Cirujano-Dentista.

1,3 Residente, Programa de PosGrado en Cirugía y Traumatología Buco-maxilo Facial de la Facultad de Odontología de Piracicaba, UNICAMP-SP . Brasil.

4 Cirujano-Dentista UNMSM, Lima- Peru. Práctica Privada.

Cirujano-Dentista. Doctor en Cirugía y Traumatología Buco-maxilo Facial. Profesor Asociado del Programa de PosGrado en Cirugía y Traumatologia Buco-maxilo Facial de la Facultad de Odontología de Piracicaba, UNICAMP-SP . Brasil.

\section{Correspondencia:}

José Luis Muñante Cárdenas

Facultad de Odontología de Piracicaba.

Departamento de Diagnóstico Oral

Área de Cirugía y Traumatología Bucomáxilo-Facial.

Av. Limeira, 901, Barrio: Areião.

CEP: 13414-903. Piracicaba -Sao Paulo. Brasil.

Fone: (19) 92953737

e-mail: jlmunante@fop.unicamp.br

Palabras clave: Fracturas mandibulares, trauma, infancia.

Key words: mandibular Fracture, trauma, childhood.

ción de la fractura, la duración de la inmovilización (Hardt 1993) ${ }^{(2)}$.

\section{Objetivos}

El presente trabajo tiene por objetivos reconocer la etiología del trauma facial e identificar los métodos de diagnóstico e indicación de tratamiento conservador en fracturas mandibulares pediátricas.

\section{Método empleado}

Se analizaron los estudios publicados en la bibliografía médica-odontoló- 
gica, utilizando los buscadores Pubmed y Medline, en inglés, español y portugués, considerando aquellas publicaciones en las que apareciesen como palabras clave "trauma", "fracture" "infancia", "fratura", "children". Se incluyeron revisiones sistemáticas, estudios prospectivos longitudinales y estudios retrospectivos, totalizando 22 publicaciones entre 1985 y el 2005, las cuales consideramos de interés para este articulo. Se excluyeron los artículos sin resumen disponible y los casos clínicos.

\section{Etiología}

Los niños sufren alrededor del 13-15\% del total de fracturas faciales con un pico de incidencia a los 10 años de edad (Qudah 2005) ${ }^{(4)}$. De éstas, el 1\% ocurren en niños de hasta 5 años y aproximadamente $8 \%$ en menores de

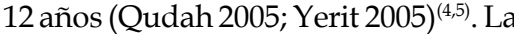
mandíbula es el hueso facial más afectado, siendo reportada su ocurrencia entre 15 a $86 \%$ del total de las fracturas faciales pediátricas (Lindahl 1987; Iida 2002; Bamjee 1996) ${ }^{(6,7,8)}$.

Las principales causas de fracturas en niños son los accidentes de tránsito los accidentes en bicicleta, las caídas de propia altura y los accidentes deportivos $^{(7)}$. Las caídas son más comunes en infantes y niños mayores de 6 años (Benoit 2000; Carroll 1987)(9,10) y los accidentes de tránsito son más comunes en los niños de 10 a 16 años, incluyéndose también fracturas por peleas o accidentes deportivos (Infante 1994; Brudvik 2000) ${ }^{(11,12)}$.

Con relación al sexo, diversos estudios revelan que los varones son más afectados que las mujeres. La proporción varones/mujeres varia desde 1:1 hasta 2.9:1 (Qudah 2005; Iida 2002; Infante 1994;McGraw 1990; Thoren 1997) $)^{(4,7,11,13,14)}$.

\section{Diagnóstico y regiones mandibulares afectadas.}

El diagnóstico de las fracturas faciales es basado en el examen clínico y confirmado mediante los exámenes imagenológicos. La historia del trauma es esencial, pues dependiendo del tipo, la intensidad y el área afectada se pueden presentar fracturas en el local o a distancia del punto de impacto (Prado) ${ }^{(20)}$

Los signos y síntomas son los mismos que en las fracturas en adultos (asimetría facial, edema de mucosa adyacente, limitación de los movimientos mandibulares, mordida abierta anterior, mal oclusión, hematoma o equimosis en el piso de boca, parestesia o anestesia en el trayecto del nervio dentario inferior) aunque la evaluación puede tornarse difícil por la poca colaboración del paciente niño (Zimmermann, 2005) ${ }^{(15)}$.

Una radiografía panorámica es de mucha utilidad y puede ser solicitada para confirmación del diagnóstico clínico (Kaban,1992) ${ }^{(19)}$. De manera complementaria, pueden ser solicitadas vistas póstero anterior, oclusal y lateral oblicua. Puede ser solicitada adicionalmente una tomografía computarizada (Zimmermann, 2005) ${ }^{(15)}$.

En lo que se refiere a la localización de las fracturas, la región condilar es la más afectada pudiendo estar presente bilateralmente en alrededor de $20 \%$ de los casos (Zimmermann, 2005) ${ }^{(15)}$. Existen algunos factores anatómicos que favorecen esta situación, tales como la alta vascularización del cóndilo pediátrico y un cuello delgado con pobre resistencia a las fuerzas de impacto durante caídas. Las fracturas de cóndilo intracapsulares son más comunes en niños menores de 6 años. En niños mayores de 6 años el sitio de fractura más común es el cuello de cóndilo (Gussack 1987) ${ }^{(17)}$, seguido por la región parasinfisiaria y por el ángulo mandibular (Qudah 2005; Iida 2002; Infante 1994; Haug 2000) $)^{(4,7,11,16)}$. A mayor edad, la región del ángulo y del cuerpo mandibular pasan a ser las regiones más afectadas (Iida 2002; Bammje 1996; Thoren 1997) ${ }^{(7,8,14)}$.

\section{Tratamiento}

\section{Tratamiento conservador}

La mandíbula infantil está sometida a una serie de particularidades que condicionan su tratamiento: hueso en crecimiento, crecimiento combinado maxilo-mandibular, presencia de gérmenes dentarios y posición más basal del nervio dentario inferior. La dinámica del crecimiento conduce a encontrarnos con diferentes situaciones en el tratamiento de estas fracturas (Hardt 1993) ${ }^{(2)}$. Factores como el gran potencial osteogénico y rápidas tasas de cicatrización en pacientes pediátricos en comparación con los adultos son de relevante importancia al considerar esta alternativa de tratamiento (Zimmermann, 2005) ${ }^{(15)}$.

Las Consideraciones generales en el tratamiento de pacientes pediátricos traumatizados deben incluir : permeabilidad de la vía aérea, balance fluido-electrolítico $\mathrm{y}$, atendiendo a su tamaño y anatomía vulnerables, un adecuado estado nutricional. En general, se acepta que la cantidad de desplazamiento de los segmentos de la fractura determinará si el tratamiento conservador es una opción adecuada. Los fragmentos óseos en los niños pueden unirse de manera parcial en 4 días y las fracturas se vuelven difíciles de reducir en 7 días, razón por la cual se debe implementar el tratamiento lo más pronto posible (Kaban 1992)(19).

Las fracturas que estén limitadas al proceso alveolar pueden ser tratadas mediante reducción cerrada e inmovilización durante dos o tres semanas. Pueden hacerse uso de splints, barras o arcos (Zimmermann, 2005) ${ }^{(15)}$. Las fracturas mandibulares sin desplazamiento y ausencia de mal oclusión son manejadas mediante observación, dieta liquida o blanda, reposo y prescripción de analgésicos. Esta conducta puede ser seguida para el tratamiento de pacientes menores de 2 años de edad con fracturas en tallo verde mínimamente desplazadas (Tanaka 1993)(22).

Cuando fuera necesario, fracturas de cuerpo, sínfisis o ángulo mandibular pueden ser fijadas e inmovilizadas a través de fijación maxilomandibular (FMM), atendiendo al tipo de fractura y al estado de desarrollo esquelético y dental del paciente (Zimmermann, 2005) $)^{(15)}$

De otro lado, existen situaciones, como en las fracturas condilares, en las que no se puede esperar una reducción anatómica del área fracturada. En estos casos, un desplazamiento pequeño o moderado resulta en función y oclusión posoperatoria adecuadas sólo si se obtuvo una correcta relación oclusal previa. Así, en fracturas condilares intracapsulares sin compromiso de la oclusión, puede optarse por un tratamiento conservador realizando una continua evaluación clínica de la oclusión y alivio sintomático del dolor. Niños menores de 10 años generalmente son tratados con fisioterapia y alivio sintomático del dolor por medio de analgésicos y antiinflamatorios (Haug 2000) ${ }^{(16)}$. En fracturas condilares extracapsulares, la FMM puede ser usada por un máximo de 2 semanas (Haug 2000; Gussack 1987) $^{(16,17)}$ continúandose luego con un periodo de rehabilitación intensivo, el cual debe ser prontamente aplicado para evitar la formación exagerada de tejido cicatricial en el área de fractura y el 
consiguiente desarrollo de anquilosis temporomandibular (Thoren 1997)(14) Estudios reportan que un manejo conservador de las fracturas condilares producen resultados satisfactorios (Choi 2005) $^{(18)}$

Fracturas de sínfisis o cuerpo mandibular en infantes total o parcialmente edéntulos pueden ser tratadas con una fijación monomandibular, la cual puede ser obtenida a través de splints acrílicos o materiales termoplásticos, pudiendo sólo ser considerada como una opción aceptable en el tratamiento de fracturas en tallo verde o en las fracturas con desplazamiento mínimo (Carroll 1987) $^{(10)}$. En fracturas que presenten un desplazamiento moderado puede ser necesaria una FMM con férulas de Gunning (Kaban 1992)(19).

Contrariamente a lo que muchos suponen, una FMM es mejor tolerada por los niños que por los adultos (Kaban 1992) ${ }^{(19)}$. Con todo, el manejo conservador tiene como desventaja absoluta una reducción anatómica limitada de la fractura y una total restricción de la función, lo cual es poco conveniente para este tipo de pacientes que ven afectada o disminuida su normal alimentación (Martinez 2004) $)^{(21)}$.

\section{Discusión}

Las fracturas faciales en niños muestran características importantes que las diferencian de las fracturas en adultos. Factores como el tamaño pequeño de la mandíbula infantil en relación al cráneo, resiliencia ósea, presencia de gérmenes dentarios, número de dientes permanentes erupcionados, la resorción de sus raíces y un posible daño al centro de crecimiento condilar mandibular, deben ser tomados en cuenta cuando tratamos este tipo de fracturas. Por estas razones, el trauma infantil debe ser objeto de especial atención ya que presentan particulares características anatómicas, fisiológicas y psicológicas propias de la edad del paciente.

Según datos reportados por Qudah ${ }^{(4)}$ e Infante ${ }^{(11)}$ la incidencia de traumas maxilofaciales se incrementa gradualmente con la edad. Esto puede explicarse por el hecho de que niños de menor edad pasan mayor tiempo protegidos por el entorno familiar, mientras que a mayor edad, el ambiente social de los niños cambia, teniendo más contacto con el mundo exterior. Los niños van a la escuela y comienzan a participar de deportes y actividades lúdicas de contacto.
Su altura, peso, fuerza y agresividad también aumentan (Iida 2002) ${ }^{(7)}$.

Durante la primera atención, una adecuada historia del trauma es esencial, pues aportará datos de especial importancia para el diagnóstico y la instauración del tratamiento inicial. El examen auxiliar de imagen solicitado para la confirmación del diagnóstico clínico es la radiografía panorámica, que es de gran utilidad, pues muestra en una sola exposición las articulaciones temporomandibulares, el ángulo, la rama, cuerpo y sínfisis (Kaban 1992) ${ }^{(19)}$. Adicionalmente, una tomografía computarizada puede ser indicada en el caso de fracturas condilares, con la finalidad de poder determinar tridimensionalmente el desplazamiento del cóndilo (Zimmermann, 2005) ${ }^{(15)}$.

Las fracturas de mandíbula deben ser tratadas a través del método más simple que produzca una adecuada reducción y estabilización. Debe tomarse en cuenta factores como la variación anatómica, rapidez de la cicatrización, grado de cooperación del paciente y potencial de interferencia del crecimiento mandibular (Kaban 1992) ${ }^{(19)}$.

La presencia de gérmenes dentarios y la elasticidad del hueso infantil fueron factores que promovieron la FMM o el esplintage como tratamiento estándar en fracturas mandibulares con dentición decidua, mientras que la reducción abierta y fijación interna rígida (FIR) eran evitadas con la finalidad de proteger los gérmenes dentários. Sin embargo, FMM puede ser difícil de conseguir en niños, principalmente debido a factores locales como la forma de las coronas de los dientes deciduos (las cuales no son retentivas), al grado de la resorción de sus raíces y a dientes permanentes anteriores parcialmente erupcionados. Todos estos factores pueden interferir para una adecuada fijación monomaxilar o intermaxilar

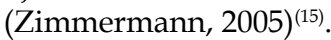

La mandíbula pediátrica es bastante maleable. Esta característica, sumada a la presencia de gérmenes dentarios bien fijos que pueden sostener -a manera de "pegamento" - los fragmentos óseos, contribuyen a que las fracturas de cuerpo y sínfisis tiendan a ser menos desplazadas y raramente conminutivas (Carroll 1987; Kaban $1992)^{(10,19)}$. Por estas razones, Kaban ${ }^{(19)}$, afirma que la mayoría de estas fracturas son pasibles de ser tratadas con reducción cerrada y FMM. Por su parte, Zimmermenn ${ }^{(15)}$, recomienda una FMM por 4 semanas, considerándola efectiva para el tratamiento de estas fracturas, incluyendo las de ángulo mandibular.

En el caso de fracturas condilares, el principal objetivo del tratamiento debe estar orientado a la restauración de la simetría mandibular, de la oclusión y de la función, sin interferir con el futuro crecimiento (Kaban 1992) ${ }^{(19)}$. Para Infantes $^{(11)}$ fracturas bajas de cuello de cóndilo deben ser tratadas inmediatamente con fijación interna rígida y la articulación temporomandibular debe ser movilizada lo más pronto posible. Aunque la reducción abierta de este tipo de fracturas ha dado buenos resultados (Infante 1994; Zimmermann, $2005)^{(11,15)}$, otros autores aún recomiendan el tratamiento conservador ( o reducción cerrada), reservando el tratamiento quirúrgico sólo para situaciones en las cuales la oclusión no haya podido ser restablecida, cuando el segmento cóndilar fue desplazado a la fosa craneal medial o cuando un cuerpo extraño esté presente (Thoren 1997; Kaban 1992)(14,19).

\section{Conclusiones}

Las causas principales de fracturas mandibulares en niños son los accidentes de tránsito y las caídas de propia altura. Estas pueden variar de acuerdo a la edad cronológica, al cambio de ambiente social y con las actividades del paciente pediátrico.

El tratamiento de las fracturas mandibulares en niños dependerá del sitio de fractura, la edad del paciente y la etapa en que se encuentre el desarrollo esquelético y dental.

Los niños poseen un alto potencial osteogénico así como altas tasas de cicatrización y reparación. Por lo tanto, una reducción anatómica en estos puede ser lograda tempranamente y los tiempos de inmovilización pueden ser más cortos.

Fracturas no desplazadas pueden ser tratadas conservadoramente mediante observación e indicaciones para una dieta blanda o líquida y administración de analgésicos cuando fuere necesario.

El manejo invasivo quirúrgico puede ser considerado cuando las técnicas cerradas tengan una alta probabilidad de mala unión o de no unión.

\section{Bibliografía}

1. Filho,E.F.A. Fracturas de mandibula: análisis de 126 casos. Ver Ass Méd Brasil. V 2000; Vol. 46: 272-276 
2. Hardt N.,Gottsauner A. The treatment of mandibular fractures in children. J Craniomaxillofac Surg. Jul 1993 21(5): $214-9$

3. Eppley BL, Prevel CD. Nonmetallic fixation in traumatic midfacial fractures. J Craniofac Surg. Mar 1997; Vol. 8(2): 103-9.

4. Qudah MA, Al-Khateeb T, Bataineh AB Rawashdeh MA. Mandibular fractures in Jordanians: a comparative study between young and adult patients. J Craniomaxillofac Surg. Apr 2005; Vol. 33(2): 103-6

5. Yerit KC, Hainich S, Enislidis G, Turhani D, Klug C, Wittwer G, Ockher M, Undt G, Kermer C, Watzinger F, Ewers R. Biodegradable fixation of mandibular fractures in children: stability and early results. Oral Surg Oral Med Oral Pathol Oral Radiol Endod. Jul 2005; Vol. 100(1): $17-24$

6. Lindahl L. Condylar fractures of the mandible. IV. Function of the masticatory system. Int J Oral Surg. Aug 1987; Vol. 6(4): 195-203

7. Iida S, Matsuya T. Paediatric maxillofacial fractures: their aetiological characters and fracture patterns.J Craniomaxillofac Surg. Aug 2002; 30(4): 237-41.

8. Bamjee Y, Lownie JF, Cleaton-Jones PE Lownie MA. Maxillofacial injuries in a group of South Africans under 18 years of age.Br J Oral Maxillofac Surg. Aug 1996; Vol. 34(4):298-302
9. Benoit R, Watts DD, Dwyer K, Kaufmann C, Fakhry S. Windows 99: a source of suburban pediatric trauma.J Trauma. Sep 2000; Vol. 49(3): 477-81; discussion 481-2

10. Carroll MJ, Mason DA, Hill CM. 'Facial fractures in children'. Br Dent J. Nov 1987; Vol. 163(9): 289

11. Infante Cossio P, Espin Galvez F, Gutierrez Perez JL, Garcia-Perla A, Hernandez Guisado JM.Mandibular fractures in children. A retrospective study of 99 fractures in 59 patients. Int J Oral Maxillofac Surg. Dec 1994; Vol. 23(6): 329-31

12. Brudvik C. Child injuries in Bergen, Norway. Injury. Dec 2000; Vol. 31(10):761-7

13. McGraw BL, Cole RR. Pediatric maxillofacial trauma. Age-related variations in injury. Arch Otolaryngol Head Neck Surg. Jan 1990; Vol. 116(1):41-5

14. Thoren H, Iizuka T, Hallikainen D, Nurminen M, Lindqvist C An epidemiological study of patterns of condylar fractures in children. Br J Oral Maxillofac Surg. Oct 1997; Vol.35(5): 306-11

15. Zimmermann CE, Troulis MJ, Kaban LB. Pediatric facial fractures: recent advances in prevention, diagnosis and management. Int J Oral Maxillofac Surg. Dec 2005; Vol. 34(8):823-33
16. Haug RH, Foss J. Maxillofacial injuries in the pediatric patient. Oral Surg Oral Med Oral Pathol Oral Radiol Endod. Aug 2000; Vol. 90(2): 126-34

17. Gussack GS, Luterman A, Powell RW, Rodgers K, Ramenofsky ML Pediatric maxilla facial trauma: unique features in diagnosis and treatment. Laryngoscope. Aug 1987; Vol. 97(8): 925-30

18. Choi J, Oh N, Kim IK. follow-up study of condyle fracture in children. Int J Oral Maxillofac Surg. Dec 2005; Vol. 34(8):851-8

19. Kaban L.B. Cirugia Oral y maxilofacial en niños. México: Editorial McGrawHill.1992.

20. Prado S. Cirugía Bucomaxilofacial. Diagnóstico e Tratamento. Brasil: Editorial Medsi. 2004

21. Martínez-Villalobos S. Osteosintesis maxilofacial con titanio. Rev Esp Cirug Oral y Maxilofacial V. Dic 2004; Vol. 26(6)

22. Tanaka N, Uchide N, Suzuki K, Tashiro T, Tomitsuka K, Kimijima Y, Amagasa T. Maxillofacial fractures in children. J Craniomaxillofac Surg. Oct 1993; Vol. 21(7): 289-93

Recibido :02-04-2008

Aceptado para publicación: 24-07-2008 